\title{
A new approach of creating a finite element stick model for flutter analysis
}

\author{
Aleš Kratochvíl1* \\ ${ }^{1}$ Dept. of Aerospace Engineering, Faculty of Mechanical Engineering, Center of Advanced \\ Aerospace Technology, Czech Technical University in Prague, Technicka Street 4, 16607, Prague 6, \\ Czech Republic.
}

\begin{abstract}
The article describes a new approach of creating a finite element stick model for the purpose of the aircraft flutter analysis. The control surface properties are merged with a lifting surface element as a special degree of freedom (DOF). The number of DOF for the coordination system is increased from the classical $6 \mathrm{DOF}$ to $9 \mathrm{DOF}$. A new beam element and concentrated mass element are present for the purpose of modal and flutter analysis. The advantage of this method lies in reducing the number of model nodes and introduction of a more appropriate arrangement of global mass matrix for eigen-value extraction. Quality of results is not affected. The paper also presents a verification example of a real sports aircraft.
\end{abstract}

\section{Flutter}

Flutter is defined as a self-excited undamped oscillation of a structure. It can occur without warning and usually leads to the destruction of an aircraft within less than a second. The flutter is caused by interaction of two or more eigen-modes together, which leads to decrease of an overall damping (structural and aerodynamic). When the damping of certain eigenmodes fall down to zero, flutter will occur.

This paper is focused on the most common type of flutter known as classical or potential flutter, which occurs in potential flow. There are also other types of flutter such as stall flutter [1], transonic flutter [2] or whirl flutter [3] which has not been considered in this paper. However, the new approach of creating a finite element stick model presented here can be used for these types of flutter as well.

The most common way to suppress flutter occurrence or increase flutter velocity is by mass balancing of the control surface. However, the current research is focus on active flutter suppression [4], [5].

An investigation of critical flutter velocity requires an airplane in final flight configuration. The input data is evaluated from the ground vibration test (GVT) and experimental determination of mass characteristics [6]. A standard process of flutter analysis is based on the structural stick resp. beam finite element model (FEM) with concentrated mass. Also a shell element model can be used. A stiffness characteristic of such a model are optimized to calculate eigen-frequencies and eigen-vector of FEM model match with ones from GVT [7]. Then an unsteady aerodynamic model is added and connected with structural model DOF. For subsonic aircraft [8] or [9] is usually used. Another way in which the flutter 
analysis can be performed is not so common in industry but it is less time consuming due to lack of structural model optimization process. This way, described in [10], uses a mathematical model derived directly in modal coordinates and works with eigen-vectors measured during GVT (not calculated from FEM model). But all aircraft configurations have to be measured during GVT because this method does not allow recalculating eigenfrequency and eigen-vector change caused by change of payload, such as standard processes do.

\section{Classical approach to creating structural model for flutter analysis}

For purpose of the standard flutter analysis, the structural stick FEM model with 6 DOF is created from the following elements presented in Fig.1:

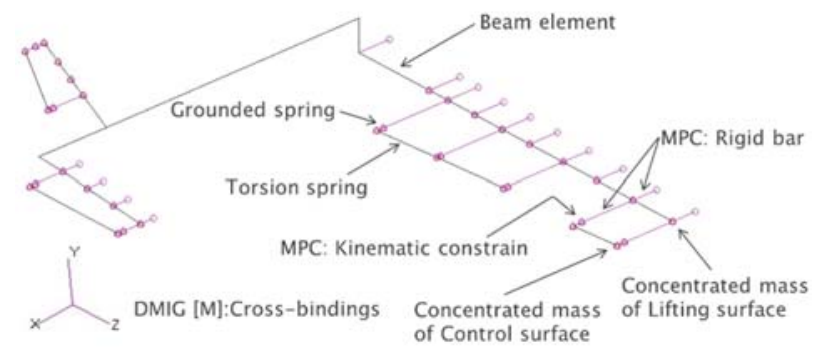

Fig. 1. Structural FEM model for classical approach.

Also it is necessary to do a direct matrix input to global mass matrix of such a model (DMGI) for the purpose of definition of cross-bindings between DOF in bending/torsion of lifting surface and rotation of control surface. These cross-bindings are given by derivation of equation of motion for oscillation airfoil by Lagrange differential equation of second kind. A derived mass matrix is formulated as

$$
[M]=\left[\begin{array}{ccc}
m & S_{Z} & \underline{S_{\delta}} \\
S_{Z} & J_{Z} & \underline{J_{\delta}+x_{b} S_{\delta}} \\
\underline{S_{\delta}} & \underline{J_{\delta}+x_{b} S_{\delta}} & J_{\delta}
\end{array}\right]
$$

where $\mathrm{m}$ is the mass of the lifting surface, $\mathrm{S}_{\mathrm{z}}$ is the static moment to elastic axis of lifting surface, $\mathrm{S}_{\delta}$ is the static moment of control surface to hinge axis, $\mathrm{J}_{\mathrm{Z}}$ is the moment of inertia to elastic axis of the lifting surface, $\mathrm{J}_{\delta}$ is the moment of inertia of control surface to hinge axis, $\mathrm{x}_{\mathrm{b}}$ is the distance between hinge axis of control surface and elastic axis of lifting surface. The cross-bindings are underlined in (1). They are outside of concentrated mass elements in global mass matrix of FEM model. Thus, they have to be defined manually via direct matrix input to specific locations of the global mass matrix, see example in Figure 2. Such as model is then optimized to results from GVT and unsteady aerodynamic model is added for the purpose of flutter analysis.

\section{New approach to creating a structural model for flutter analysis}

A new approach of creating the FEM stick model is based on merging a property of control surface elements to the elements of lifting surface. It can be done by extension of the global 

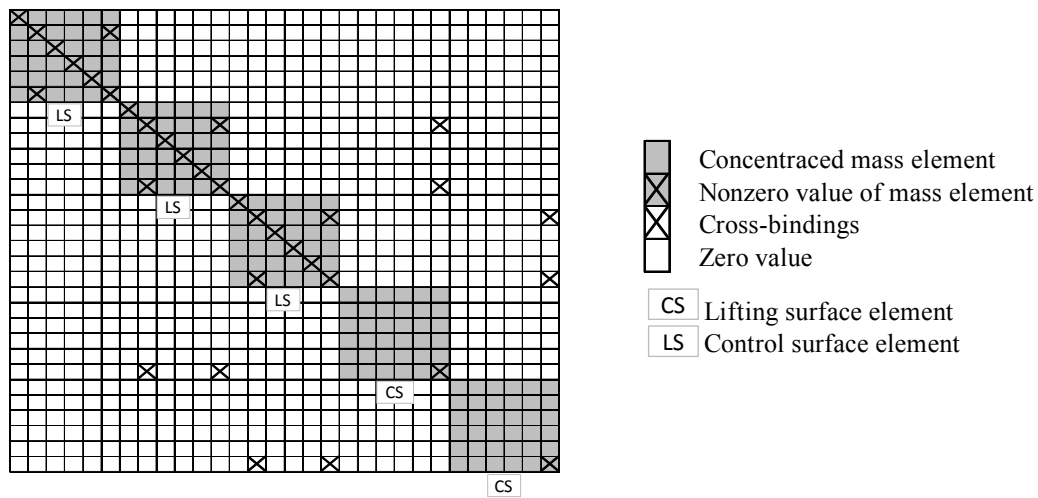

CS Lifting surface element

LS Control surface element

Fig. 2. Example of global mass matrix.

coordinate system from standard 6DOF to 9DOF. The new coordinate system, see Figure 3, contains three DOF for translation of lifting surface X,Y,Z three DOF for rotation of lifting surface RX, RY, RZ and another three DOF for control surface rotation RXX, RYY, RZZ. Where RZZ DOF is intended to be used for control surface rotation. The other two have no physical meaning thus their properties will be zero in the local matrix. They are present here for the purpose of transforming an element from local to global coordinate system only.

Using this approach, there is no need for a definition of the node, concentrated mass and torsion spring, nor kinematic constraint especially for the control surface. Only the grounded spring still has to be defined. The structural FEM model for the new approach is presented in Figure 3.

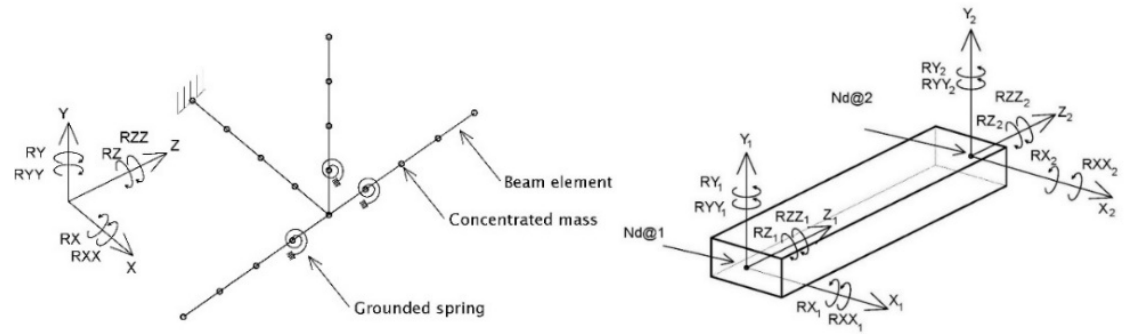

Fig. 3. Structural FEM model for new approach.

Fig. 4. Beam element.

$$
\left[K_{E m}\right]=\left[\begin{array}{cccccccccccccccccc}
\frac{12 E I_{y}}{l^{3}} & 0 & 0 & 0 & \frac{6 E I_{y}}{l^{2}} & 0 & 0 & 0 & 0 & -\frac{12 E I_{y}}{l^{3}} & 0 & 0 & 0 & \frac{6 E I_{y}}{l^{2}} & 0 & 0 & 0 & 0 \\
0 & \frac{12 E I_{x}}{l^{3}} & 0 & -\frac{6 E I_{x}}{l^{2}} & 0 & 0 & 0 & 0 & 0 & 0 & -\frac{12 E I_{x}}{l^{3}} & 0 & -\frac{6 E I_{x}}{l^{2}} & 0 & 0 & 0 & 0 & 0 \\
0 & 0 & \frac{E A}{l} & 0 & 0 & 0 & 0 & 0 & 0 & 0 & 0 & -\frac{E A}{l} & 0 & 0 & 0 & 0 & 0 & 0 \\
0 & -\frac{6 E I_{x}}{l^{2}} & 0 & \frac{4 E I_{x}}{l} & 0 & 0 & 0 & 0 & 0 & 0 & \frac{6 E I_{x}}{l^{2}} & 0 & \frac{2 E I_{x}}{l} & 0 & 0 & 0 & 0 & 0 \\
\frac{6 E I_{y}}{l^{2}} & 0 & 0 & 0 & \frac{4 E I_{y}}{l} & 0 & 0 & 0 & 0 & -\frac{6 E I_{y}}{l^{2}} & 0 & 0 & 0 & \frac{2 E I_{y}}{l} & 0 & 0 & 0 & 0 \\
0 & 0 & 0 & 0 & 0 & \frac{G J_{P}}{l} & 0 & 0 & 0 & 0 & 0 & 0 & 0 & 0 & -\frac{G J_{P}}{l} & 0 & 0 & 0 \\
0 & 0 & 0 & 0 & 0 & 0 & 0 & 0 & 0 & 0 & 0 & 0 & 0 & 0 & 0 & 0 & 0 & 0 \\
0 & 0 & 0 & 0 & 0 & 0 & 0 & 0 & 0 & 0 & 0 & 0 & 0 & 0 & 0 & 0 & 0 & 0 \\
0 & 0 & 0 & 0 & 0 & 0 & 0 & 0 & \frac{k_{\delta T}}{l} & 0 & 0 & 0 & 0 & 0 & 0 & 0 & 0 & -\frac{k_{\delta T}}{l} \\
\frac{12 E I_{y}}{l^{3}} & 0 & 0 & 0 & -\frac{6 E I_{y}}{l^{2}} & 0 & 0 & 0 & 0 & \frac{12 E I_{y}}{l^{3}} & 0 & 0 & 0 & -\frac{6 E I_{y}}{l^{2}} & 0 & 0 & 0 & 0 \\
0 & -\frac{12 E I_{x}}{l^{3}} & 0 & \frac{6 E I_{x}}{l^{2}} & 0 & 0 & 0 & 0 & 0 & 0 & \frac{12 E I_{x}}{l^{3}} & 0 & \frac{6 E I_{x}}{l^{2}} & 0 & 0 & 0 & 0 & 0 \\
0 & 0 & -\frac{E A}{l} & 0 & 0 & 0 & 0 & 0 & 0 & 0 & 0 & \frac{E A}{l} & 0 & 0 & 0 & 0 & 0 & 0 \\
0 & -\frac{6 E I_{x}}{l^{2}} & 0 & \frac{2 E I_{x}}{l} & 0 & 0 & 0 & 0 & 0 & 0 & \frac{6 E I_{x}}{l^{2}} & 0 & \frac{4 E I_{x}}{l} & 0 & 0 & 0 & 0 & 0 \\
\frac{6 E I_{y}}{l^{2}} & 0 & 0 & 0 & \frac{2 E I_{y}}{l} & 0 & 0 & 0 & 0 & -\frac{6 E I_{y}}{l^{2}} & 0 & 0 & 0 & \frac{4 E I_{y}}{l} & 0 & 0 & 0 & 0 \\
0 & 0 & 0 & 0 & 0 & -\frac{G J_{P}}{l} & 0 & 0 & 0 & 0 & 0 & 0 & 0 & 0 & \frac{G J_{P}}{l} & 0 & 0 & 0 \\
0 & 0 & 0 & 0 & 0 & 0 & 0 & 0 & 0 & 0 & 0 & 0 & 0 & 0 & 0 & 0 & 0 & 0 \\
0 & 0 & 0 & 0 & 0 & 0 & 0 & 0 & 0 & 0 & 0 & 0 & 0 & 0 & 0 & 0 & 0 & 0 \\
0 & 0 & 0 & 0 & 0 & 0 & 0 & 0 & -\frac{k_{\delta T}}{l} & 0 & 0 & 0 & 0 & 0 & 0 & 0 & 0 & \frac{k_{\delta T}}{l}
\end{array}\right]
$$


There were developed new elements for this approach, which correspond to the 9DOF coordinate system. The first one is massless beam element shown in Figure 4. Which is based on CBAR element with two nodes. The local stiffness matrix of an element is given by equation (2). Where $\mathrm{E}$ and $\mathrm{G}$ stand for Young's and shear modulus respectively. $\mathrm{I}_{\mathrm{x}}, \mathrm{I}_{\mathrm{y}}$ are area moment of inertia in bending, and in-plane bending, $\mathrm{J}_{\mathrm{p}}$ is torsional constant, A is area of bar cross section, 1 denotes length of the element and $\mathrm{k}_{\delta \mathrm{T}}$ stands for torsional stiffness of control surface.

$[M]=\left[\begin{array}{ccccccccc}m & 0 & 0 & 0 & 0 & 0 & 0 & 0 & 0 \\ 0 & m & 0 & 0 & 0 & S_{Z} & 0 & 0 & S_{\delta} \\ 0 & 0 & m & 0 & 0 & 0 & 0 & 0 & 0 \\ 0 & 0 & 0 & J_{x} & 0 & 0 & 0 & 0 & 0 \\ 0 & 0 & 0 & 0 & J_{y} & 0 & 0 & 0 & 0 \\ 0 & S_{Z} & 0 & 0 & 0 & J_{z} & 0 & 0 & C B C \\ 0 & 0 & 0 & 0 & 0 & 0 & 0 & 0 & 0 \\ 0 & 0 & 0 & 0 & 0 & 0 & 0 & 0 & 0 \\ 0 & S_{\delta} & 0 & 0 & 0 & C B C & 0 & 0 & J_{\delta}\end{array}\right] \quad(3) \quad[K]=\left[\begin{array}{ccccccccc}0 & 0 & 0 & 0 & 0 & 0 & 0 & 0 & 0 \\ 0 & 0 & 0 & 0 & 0 & 0 & 0 & 0 & 0 \\ 0 & 0 & 0 & 0 & 0 & 0 & 0 & 0 & 0 \\ 0 & 0 & 0 & 0 & 0 & 0 & 0 & 0 & 0 \\ 0 & 0 & 0 & 0 & 0 & 0 & 0 & 0 & 0 \\ 0 & 0 & 0 & 0 & 0 & 0 & 0 & 0 & 0 \\ 0 & 0 & 0 & 0 & 0 & 0 & 0 & 0 & 0 \\ 0 & 0 & 0 & 0 & 0 & 0 & 0 & 0 & 0 \\ 0 & 0 & 0 & 0 & 0 & 0 & 0 & 0 & k_{\delta}\end{array}\right]$

The second element is concentrated mass, which comes from equation (1). The local mass matrix is defined by equation (3) where $\mathrm{CBC}$ is given as

$$
C B C=J_{\delta}+x_{b} S_{\delta}
$$

The last element is a grounded spring represented by local stiffness matrix as given in equation (5). Where $\mathrm{k}_{\delta}$ is the stiffness of control.

Fig. 5. Deformation visualisation.

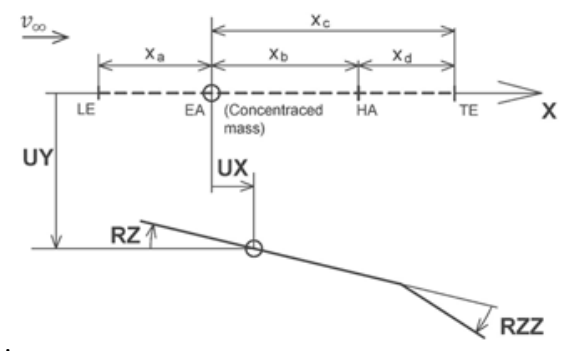

A visualisation of model deformation, can be done from the calculated eigen-vector and equations $(6) \div(9)$, which can be automatically generated for each node of the model. The equations can be used similarly for in-plane or side deformation of node.

$$
\begin{array}{lll}
y_{L E}=U Y+\sin (R Z)\left(-x_{a}\right) & \text { (6) } & y_{H A}=U Y+\sin (R Z) x_{b} \\
y_{E A}=U Y & \text { (7) } & y_{T E}=U Y+\sin (R Z) x_{c}+\sin (R Z Z) x_{d}
\end{array}
$$

Where index LE means leading edge, TA is for trailing edge. The meaning of $\mathrm{x}_{\mathrm{a}}$ is distance between elastic axis and leading edge, $x_{d}$ is control surface chord. UY, RZ, RZZ are appropriate components of calculated eigen-vectors in vertical translation, torsion and control surface rotation respectively. Figure 5 shows the node deformation cross-section.

\section{Verification example}

A tail-plane of a motor glider UMF-13 Lambada was used as a verification example. The modal parameters were determined by the ground vibration test and mass characteristic was experimentally measured [11]. The verification example was calculated in MSC.Nastran software with the classical approach with 6DOF coordinate system, and by the new approach with 9DOF coordinate system in Matlab. The airplane flutter analysis was also carried out by the model, which works in modal coordinates with directly imported eigen-shapes from 
GVT, by in-house software Flutter 3D - 2.0, developed at Department of Aerospace Engineering, Faculty of Mechanical Engineering, Czech Technical University in Prague [10]. The comparison of structural FEM models for Nastran and Matlab is presented in Table 1.

Both models have exactly the same position of nodes and the input values of stiffness and mass characteristics are the same as well. The visualisation of both models is in Figures 6, 7. Boundary conditions - fixation of free fuselage end is applied for both models.

Table 1. FEM models comparison.

\begin{tabular}{|c|c|c|}
\hline Component & $\begin{array}{c}\text { Nastran } \\
\text { 6DOF }\end{array}$ & $\begin{array}{c}\text { Matlab } \\
\text { 9DOF }\end{array}$ \\
\hline Independent nodes & 70 & 42 \\
\hline Dependent nodes & 112 & 0 \\
\hline Beam elements & 41 & 41 \\
\hline Concentrated mass elements & 70 & 42 \\
\hline
\end{tabular}

\begin{tabular}{|c|c|c|}
\hline Component & $\begin{array}{c}\text { Nastran } \\
\text { 6DOF }\end{array}$ & $\begin{array}{c}\text { Matlab } \\
\text { 9DOF }\end{array}$ \\
\hline DMIG entry & 28 & 0 \\
\hline Torsion spring elements & 27 & 0 \\
\hline Grounded spring elements & 2 & 2 \\
\hline Multi-point constrain & 140 & 0 \\
\hline Visualisation equations & 0 & 441 \\
\hline
\end{tabular}
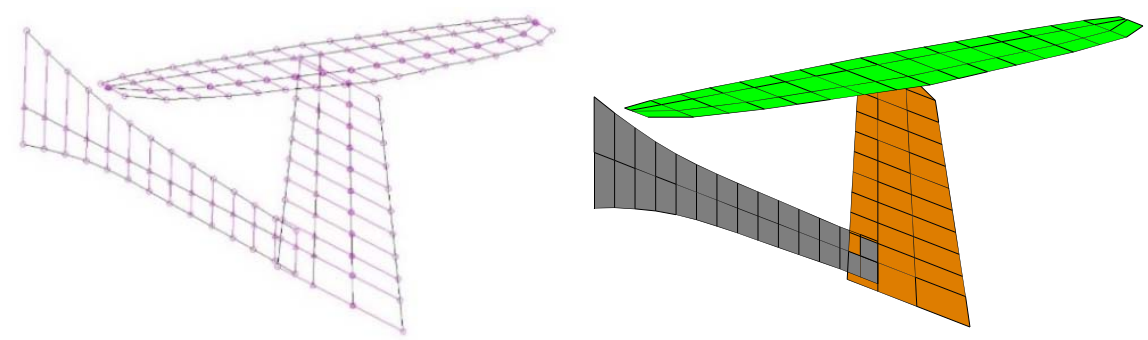

Fig. 6. Nastran 6DOF model visualisation.

Fig. 7. Matlab 9DOF model visualisation.

\subsection{Modal analysis}

Table 2 presents a list of eigen-frequencies after the optimization process. An Enhance Invers Power algorithm for eigen-value extraction in Nastran and a QZ algorithm for Matlab was used.

The eigen-vectors are compared by Modal Assurance Criterion with excellent match, see Figure 8. From eigen-frequencies comparison of 9DOF model with GVT in last column of Table 2 it can be seen that the dynamical similarity is quite good. Only for the $14^{\text {th }}$ mode, the optimization process was not successful. However, this mode has no influence on flutter velocity of the analysed airplane.

Table 2. Eigen-frequencies comparison.

\begin{tabular}{|c|c|c|c|c|c|c|}
\hline \multirow{2}{*}{ Mode } & \multirow{2}{*}{ Eigen-shape } & \multicolumn{3}{|c|}{ Eigen-frequency [Hz] } & \multirow{2}{*}{$\begin{array}{l}\text { Difference } \\
\text { 6DOF vs } \\
\text { 9DOF [\%] }\end{array}$} & \multirow{2}{*}{$\begin{array}{l}\text { Difference } \\
\text { 9DOF vs } \\
\text { GVT [\%] }\end{array}$} \\
\hline & & $\begin{array}{l}\text { Nastran } \\
\text { 6DOF }\end{array}$ & $\begin{array}{l}\text { Matlab } \\
\text { 9DOF }\end{array}$ & GVT & & \\
\hline 1. & Elevator rotation & 4,92 & 4,93 & 4,91 & 0,1 & 0,3 \\
\hline 2. & $1^{\text {tt }}$ Fuselage side bending & 5,10 & 5,09 & 5,06 & 0,3 & 0,5 \\
\hline 3. & $1^{\text {tt }}$ Fuselage torsion & 8,09 & 8,11 & 8,50 & 0,2 & 4,8 \\
\hline 4. & $1^{\text {st }}$ Fuselage vertical bending & 9,18 & 9,18 & 9,19 & 0,1 & 0,1 \\
\hline 5. & Rudder rotation & 10,59 & 10,90 & 10,90 & 3,0 & 0,0 \\
\hline 6. & Fin torsion & 14,91 & 14,68 & 14,90 & 1,6 & 1,5 \\
\hline 7. & Fin bending & 20,79 & 20,60 & 19,20 & 1,0 & 6,8 \\
\hline 8. & $1^{\text {th }}$ Symmetric stabilizer bending & 25,01 & 24,85 & 25,10 & 0,6 & 1,0 \\
\hline 9. & $1^{\text {st }}$ Elevator torsion & 25,90 & 25,80 & 25,50 & 0,4 & 1,2 \\
\hline 10. & $2^{\text {nd }}$ Elevator torsion & 37,27 & 37,28 & N/A & 0,0 & - \\
\hline 11. & $2^{\text {nd }}$ Vertical fuselage bending & 42,43 & 42,59 & N/A & 0,4 & - \\
\hline 12. & Antisymmetric stabilizer torsion & 55,02 & 54,92 & 57,80 & 0,2 & 5,2 \\
\hline 13. & Symmetric stabilizer torsion & 63,23 & 63,26 & 57,00 & 0,1 & 9,9 \\
\hline 14. & 2nd Fuselage side bending & 66,36 & 66,38 & 17,00 & 0,0 & 74,4 \\
\hline 15. & $1^{\text {st }}$ Antisymmetric stabilizer bending & 71,01 & 70,97 & 77,80 & 0,1 & 9,6 \\
\hline
\end{tabular}




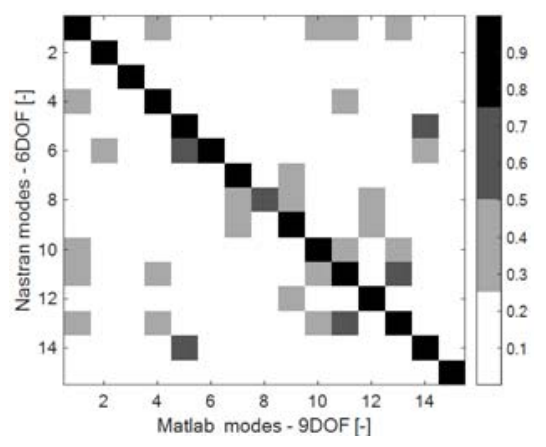

Fig. 8. MAC matrix

By comparing eigen-frequencies of FEM models to each other in penultimate column, the frequencies are almost the same. The difference is in order of tenths of percent, except modes $5^{\text {th }} \div 7^{\text {th }}$ where the difference is in order of unit of percent. It was verified that this frequency difference is not caused by a different eigen-value extraction method. The Nastran global stiffness and mass matrix were imported to Matlab and solved by QZ algorithm. The calculated eigen-frequency was the same as from Nastran with Enhance Invers Power algorithm.

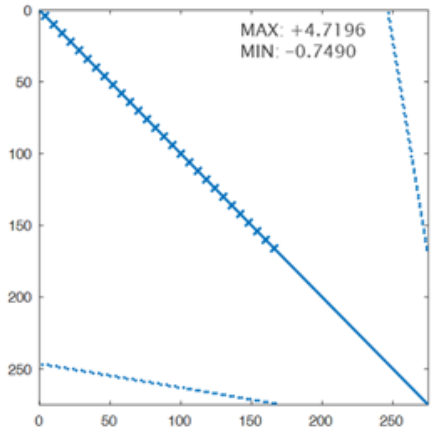

Fig. 9. Global mass matrix 6DOF model

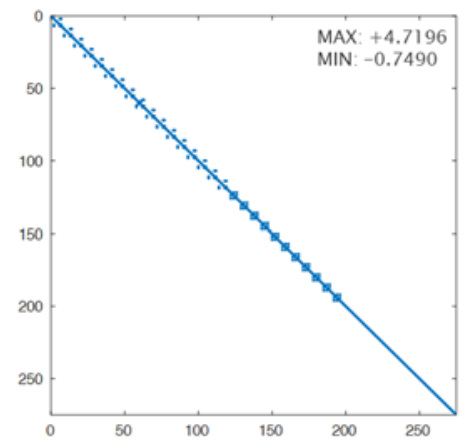

Fig. 11. Global mass matrix 9DOF model

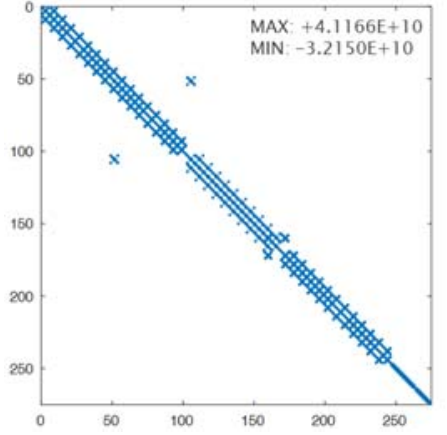

Fig. 10. Global stiffness matrix 6DOF model

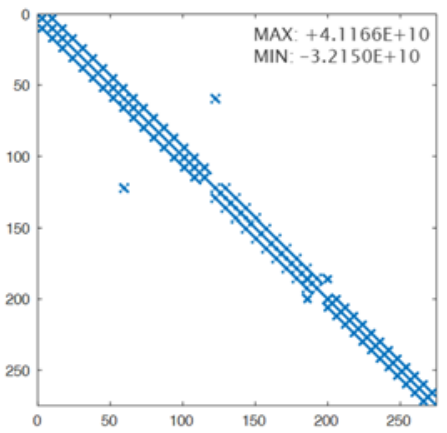

Fig. 12. Global stiffness matrix 9DOF model

This difference could be caused by the global matrix arrangement. The arrangement of global matrix for 6DOF and 9DOF models with applied boundary conditions with deleted zeros row and column are in Figures $9 \div 12$. The size of each matrix is $274 \times 274$. It can be seen that mass matrix differ in arrangement of cross-biding values of lifting surface and control surface, which are for the 9DOF model situated very close to the diagonal. This arrangement is quite useful from a numerical point of view, because the matrix inversion algorithm during eign-value extraction process can be simplified for a band matrix. The difference in stiffness matrix lies in position of control surface torsion stiffness. It is situated 
on the end for 6DOF model, while for 9DOF model is distributed along the diagonal. The values of all matrices are the same, they differ in positions of values only. A condition number and CPU time for eigen-value calculation of matrix [A] according the equation (10), carried out by QZ algorithm in Matlab for both models are present in Table 3.

$$
A=\left[\begin{array}{cc}
0 & I \\
-M \backslash K & 0
\end{array}\right]
$$

Table 3. Comparison of eigen-value calculation.

\begin{tabular}{|c|c|c|}
\hline Component & Nastran 6DOF & Matlab 9DOF \\
\hline Condition number [-] & $2,05 \mathrm{E}+13$ & $2,03 \mathrm{E}+13$ \\
\hline CPU Time [sec] & 0,59 & 0,06 \\
\hline
\end{tabular}

While the conditions numbers differ just about $1 \%$ the CPU time differ in order in disadvantage of the 6DOF model. Both times are imperceptible for humans. However, it can be of non-negligible advantage for flutter analysis with added unsteady aerodynamic matrices, repetition process of eigen-value extraction for each velocity step and an iteration process of eigen-value extraction for each mode, in the case of the most popular flutter analysis method in industry PK-method.

\subsection{Flutter analysis}

The Theodorsen unsteady aerodynamic model also known as Strip theory was used for the purpose of flutter analysis. The 29 strips were used. Only the Flutter 3D -2.0 works with 200 strips. The geometry of each strip is the same for the 6DOF and 9DOF model.

Table 4. Flutter summary.

\begin{tabular}{|c|c|c|c|}
\hline Component & Nastran 6DOF & Matlab 9DOF & Flutter 3D - 2.0 \\
\hline Flutter velocity $[\mathrm{km} / \mathrm{h}]$ & 197,5 & 191,6 & 196,0 \\
\hline Flutter frequency $[\mathrm{Hz}]$ & 8,10 & 8,14 & 7,36 \\
\hline Condition number $[-]$ & $1,33 \mathrm{E}+14$ & $2,00 \mathrm{E}+13$ & N/A \\
\hline CPU Time $[\mathrm{sec}]$ & 4,5 & 3,5 & 67,7 \\
\hline Method & PK- & P- & K- \\
\hline
\end{tabular}

The flutter analysis for the 6DOF model in Nastran was performed using PK-Method, Theodorsen function was solved by Bessel function with 16 reduced frequencies in the range $0,08 \div 26,7$ [-] and using SPLINE2 and SPLINE3 for connection structural and aerodynamic model. The flutter analysis for the 9DOF model in Matlab was performed using P-Method, described in [5]. The Theodorsen function was not solved by this method, instead a Duhamel integral of Wagner function in feedback loop is calculate. The Flutter $3 \mathrm{D}-2.0$ is using the modified K-method with iteration schema for obtaining the eigen-values from flutter matrix. The Theodorsen function was also solved by the Bessel function. For all three models a compressibility effect and structural damping was not considered. Analysed density is 1,225 $\mathrm{kg} . \mathrm{m}^{-3}$. Flutter velocities and frequencies are listed in Table 4. Flutter is caused by coupling modes $1^{\text {st }}$ Vertical fuselage bending and Elevator rotation. The difference in flutter velocities is $6,1 \mathrm{~km} / \mathrm{h}$ i.e. $3 \%$. The condition number of the flutter matrix differs in order, in benefit of the 9DOF model. The CPU time is for complete calculation, not only eigen-value extraction, at the velocity $40 \mathrm{~m} / \mathrm{s}$, by listed software and method. Thus, listed CPU time is not properly comparable due to using different software, different method of building the flutter matrix and different methods of flutter analysis. The PK-method in Nastran uses iteration scheme instead of P-method, which does not.

\section{Conclusion}


The new approach for creating the stick structural model for flutter analysis was present together with the new FEM elements. The method gives less calculation time and less condition number. It is caused by global mass matrix arrangement, where cross binding values are much closer to the diagonal than for the classical approach. Thus, the eigen-value calculation algorithm is faster. The calculated eigen-frequencies and flutter velocity are with negligible difference.

Authors acknowledge support from the ESIF, EU Operational Programme Research, Development and Education, and from the Center of Advanced Aerospace Technology.

\section{References}

1. W. G. Luber, J. Becker, Buffeting and single degree of freedom flutter at transonic speeds, 36th AIAA Applied Aerodynamics Conference, AIAA 2018-3643, (2018) ISBN: 978-162410559-3, DOI: 10.2514/6.2018-3643.

2. H. Güner, D. Thomas, G. Dimitriadis, V. Terrapon, Unsteady aerodynamic modeling methodology based on dynamic mode interpolation for transonic flutter calculations, Journal of Fluids and Structures, Vol. 84, pp. 218-232, (2019) ISSN: 08899746, DOI:10.1016/j.jfluidstructs.2018.11.002.

3. J. Čečrdle, Whirl flutter optimisation-based solution of twin turboprop aircraft using a full-span model, Applied and Computational Mechanics, Vol.11, Issue 1, pp.5-22, (2017), ISSN: 1802-608, e-ISSN:2336-1182, DOI: 10.24132/acm.2017.324.

4. F. Svoboda, M. Hromčík, Active flutter suppression by means of fixed-order Hळ control: Results for the benchmark active control technology (BACT) wing, 18th European Control Conference, Article number: 8795733, pp. 119-124, (2019) ISBN: 978390714400-8, DOI: 10.23919/ECC.2019.8795733.

5. A. Kratochvíl, F. Svoboda, T. Sommer, S. Slavík, Design for active flutter suppression and model verification. International Scientific Congress Innovations in Engineering: Scientific-technical union of mechanical engineering, pp. 47-50, (2016), ISSN 13103946, 2016.

6. J. Čečrdle, V. Hlavatý, Aeroelastic Analysis of Light Sport Aircraft Using Ground Vibration Test Data, Proceedings of the Institution of Mechanical Engineers, Part G: Journal of Aerospace Engineering, Vol. 229, Issue 12, pp. 2282-2296, (2015), ISSN 0954-4100, e-ISSN 2041-3025, DOI: 10.1177/0954410015573557.

7. J. Čečrdle, Updating of finite element model of aircraft structure according results of ground vibration test, Proceedings of the Institution of Mechanical Engineers, Part G: Journal of Aerospace Engineering, Vol. 230, Issue 7, pp. 1348-1356, (2016), ISSN 09544100, DOI: 10.1177/0954410015608887.

8. A. Edward, W. P. Rodden, A doublet-Lattice Method for Calculating Lift Distributions on Oscillating Surfaces in Subsonic Flows, AIAA Journal, Vol. 7, Issue 2, pp. 279-285, (1969), ISSN: 00011452.

9. T. Theodorsen, I. E. J. Garrick, Nonstacionary Flow a Wing-AileronTab Combination Including Aerodynamic Balance, NACA Technical Report, No. 736, (1943).

10. S. Slavík, K. Weigl, Flutter Calculation Model with Isolated Modal Characteristics of Control Surfaces for Small Sport Airplanes, Czech Aerospace Proceedings, No. 2/2008, pp. 44-49, (2008), ISSN 1211-877X.

11. A. Kratochvil, T. Sommer, S. Slavík, Flutterová analýza letounu UMF-13 Lambáda s rozpětím 15m. Technical Report, Czech Technical University in Prague, Faculty of Mechanical Engineering, Department of Aerospace Engineering, Report no. TZP/ULT/7/2014, (2015) 